\title{
Impact on mortality following first acute myocardial infarction of distance between home and hospital: cohort study
}

\author{
L Wei, ${ }^{1}$ C C Lang, ${ }^{2}$ F M Sullivan, ${ }^{3}$ P Boyle, ${ }^{4}$ J Wang, ${ }^{1}$ S D Pringle, ${ }^{2}$ T M MacDonald
}

\begin{abstract}
${ }^{1}$ Medicines Monitoring Unit (MEMO), Division of Medicine and Therapeutics, Ninewells Hospital and Medical School, Dundee, UK; ${ }^{2}$ Division of Medicine and Therapeutics, Ninewells Hospital and Medical School, Dundee, UK; ${ }^{3}$ Tayside Centre for General Practice, Division of Community Health Sciences, Dundee, UK; ${ }^{4}$ School of Geography and Geosciences, University of St Andrews, St Andrews, UK
\end{abstract}

\section{Correspondence to:}

Professor Thomas M MacDonald, Medicines Monitoring Unit, Division of Medicine and Therapeutics, Ninewells Hospital and Medical School, Dundee DD1 9SY, UK; m.macdonald@ dundee.ac.uk

Accepted 9 October 2007 Published Online First

5 November 2007

\section{ABSTRACT \\ Objective: To investigate the effect of distance between home and acute hospital on mortality outcome of patients experiencing an incident myocardial infarction (MI).}

Design: Cohort study using a record linkage database. Setting: Tayside, Scotland, UK.

Patients: 10541 patients with incident acute MI between 1994 and 2003 were identified from Tayside hospital discharge data and from death certification data. Main outcome measures: Ml mortality in the community, all-cause mortality in hospital and all-cause mortality during follow-up.

Results: 4133 subjects died following incident $\mathrm{MI}$ in the community (that is, were not hospitalised), 6408 patients survived to be hospitalised and 1010 of these (15.8\%) died in hospital. Of 5398 discharged from hospital, 1907 $(35.3 \%)$ died during a median of 3.2 years of follow-up. After adjustment for rurality and other known risk factors, distance between home and admitting hospital was significantly associated with increased mortality both before hospital admission (adjusted odds ratio (OR), 2.05, $95 \% \mathrm{Cl} 1.00$ to 4.21 for $>9$ miles and $1.46,1.09$ to 1.95 for 3-9 miles when compared to $<3$ miles) and after hospitalisation (adjusted hazard ratio (HR) 1.90, 1.19 to 3.02 and $1.27,0.96$ to 1.68$)$. However, there was no effect of distance on in-hospital mortality (adjusted OR $0.95,0.45$ to 2.03 and $1.02,0.66$ to 1.58 ).

Conclusion: The distance between home and hospital of admission may predict mortality in subjects experiencing a first acute Ml. This association was found both before and after hospitalisation. Further studies are needed to explore the reasons for this association. However these data provide support for policies that locate services for acute $\mathrm{Ml}$ closer to where patients live.

Geographical inequalities in care in the UK National Health Service (NHS) may relate to the physical distance between patients and NHS facilities. In Scotland, official policy in the government's response to the Kerr report, supports the greater provision of acute services in the community. ${ }^{12}$ Geographical inequality for patients with myocardial infarction (MI) may be related to time to thrombolysis as it is established that early thrombolysis and cardiopulmonary resuscitation improve the mortality outcome. ${ }^{34}$ Geographical inequality could also be explained by more stoical behaviour of subjects in a rural setting or other factors. We have done a large population-based study to investigate the effect of distance between home and acute hospital on mortality outcome of patients experiencing an incident MI.

\section{METHODS}

The study was carried out in Tayside, Scotland, using the Medicines Monitoring Unit's recordlinkage database. The database covers a population of approximately 400000 within a geographical area of approximately 4600 square miles. The data collection methods for this database have previously been described..$^{5}$ In brief, it contains several datasets including all dispensed community prescriptions, hospital discharge data, biochemistry results and other datasets that are linked by a unique patient identifier, the community health number. Diagnoses have been validated by inspection of the general practitioner (GP) records. ${ }^{6}$ These data are anonymised for the purposes of research. The project was approved by the Tayside Caldicott Guardians who are appointed by the government to protect the confidentiality of medical records and the Tayside committee on research medical ethics.

\section{Patient population}

The study population included subjects who were resident in Tayside and registered with a GP in January 1994 and remained in Tayside until December 2003 or died during the study perioda fixed population $(n=347$ 131). Study subjects were patients experiencing an incident MI who had not previously been hospitalised with a diagnosis of acute MI between January 1994 and December 2003.

\section{Prehospital coronary mortality}

We studied patients who died out-of-hospital with a primary certified cause of death (data from the General Register Office for Scotland) of MI (ICD-9 code 410 and ICD-10 code I21).

Patients hospitalised with $\mathrm{MI}$ and follow-up mortality Patients admitted to Tayside hospitals with their first MI were identified from the Tayside hospital discharge data using the primary diagnosis ICD-9 code of 410 and ICD-10 code I21. We tracked the all-cause mortality of patients during their hospital stay and following discharge from hospital.

Distance between home and hospital of admission Distance between home and hospital of admission was calculated based on the grid reference of the postcode of the patient's address and the grid reference of the address of the admitting hospital. For patients who died outside hospital the grid reference of their nearest acute hospital was used
This paper is freely availab online under the BMJ Jounal unlocked scheme, see http:/ heart.bmi.com/info/unlocked.dt 


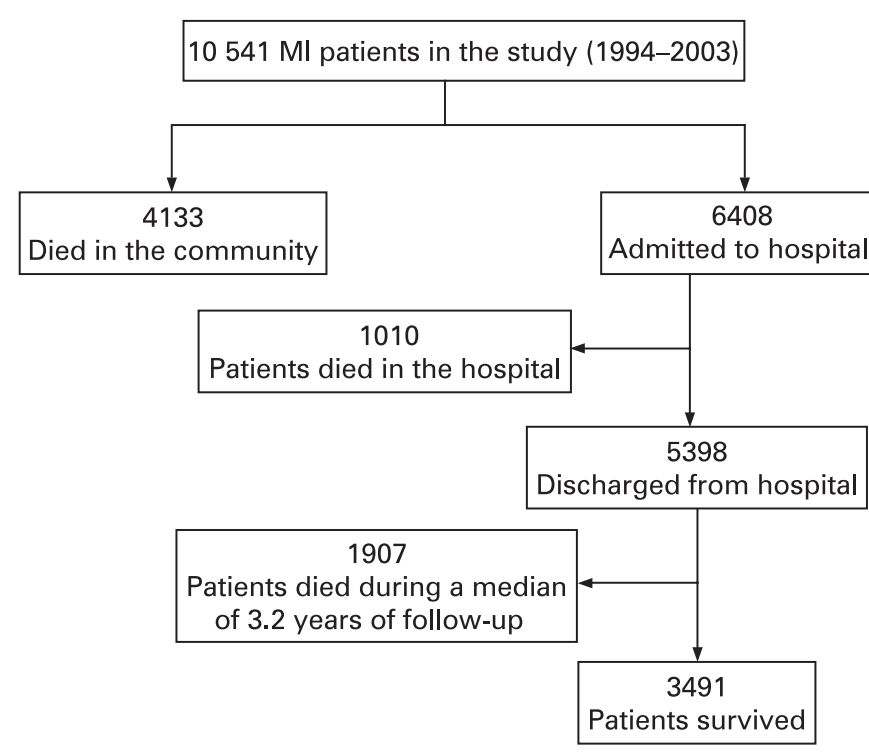

Figure 1 Flow chart of patients in the study.

to calculate the distance between home and the closest possible hospital of admission. The thrombolytic treatment strategy for Tayside during the study period was in-hospital initiation of treatment. About half of the patients lived within 5 miles $(8 \mathrm{~km})$ from these hospitals and $98.5 \%$ patients lived within 25 miles. The distance between home and hospital was thus categorised into tertiles: (1) distance $<3$ miles; (2) distance between 3-9 miles; (3) distance $>9$ miles. We obtained a rurality code from patient postcodes (that is, urban and rural classification of postcodes for Scotland, a code of 1 means large urban area with settlement of over 125000 people and a code of 8 means very remote rural area with settlement of fewer than 3000 people with a drive time of over 60 minutes to a settlement of 10000 or more). ${ }^{7}$ We also calculated the travel time by car along the road network, the car speed being based on the speed limit for the different types of road.

\section{Thrombolysis data}

Three major hospitals (Ninewells Hospital, Perth Royal Infirmary and Stracathro Hospital) in Tayside provided acute services. All these hospitals had coronary care units and accredited cardiologists working in them. However, catheter laboratory facilities were available only at Ninewells Hospital. In-hospital thrombolysis data were available for Ninewells Hospital between December 2001 and December 2004 $(n=366)$. We used these data in a subgroup analysis of patients to access the impact of thrombolysis on outcome.

\section{Postmortem examination for community MI death}

Data were obtained from the General Register Office for those patients who had been certified as an MI death in the community and who also had a subsequent postmortem examination. These subjects were more likely to be those in whom there was some diagnostic uncertainty and many of these post mortems were carried out for medicolegal reasons.

\section{Study outcome}

The outcome was the individual components: prehospital MI mortality, in-hospital all-cause mortality and follow-up allcause mortality until 31 December 2003.

\section{Statistical analysis}

Patients' characteristics were summarised as mean (SD) for continuous variables and number of subjects (\%) for categorical variables. The $\chi^{2}$ and $t$ tests were performed to determine significant differences between dead and alive patients. The Scottish 2000 standard population was used to calculate the agestandardised mortality rates. A logistic regression model was used to estimate the effects of distance between home and hospital of admission for both prehospital mortality and in-hospital mortality. Survival analysis was used to estimate the effects of distance between home and hospital of admission for post-hospital mortality since patients had different follow-up times. The results were adjusted for demographic information including age, gender, Carstairs deprivation score (derived from the patients' postcode and census data comprising social class, overcrowding, male unemployment and non-car ownership ${ }^{8}$ ), other covariates including day of week of admission, ${ }^{9}$ diabetes mellitus, and cardiovascular drug use (lipid-lowering drugs, antiplatelet drugs, $\beta$-blockers, angiotensin-converting enzyme (ACE) inhibitors, diuretics, nitrates, $\boldsymbol{\alpha}$-blockers, calcium blockers), interactions between distance and social deprivation or gender and estimated travel time and rurality. All statistical analyses were carried out using SAS version 8.2 (SAS institute, Cary, NC, USA).

\section{RESULTS}

This study included 10541 patients (fig 1). Table 1 shows the characteristics of the patients. The estimated median travel times by car from home and admitted hospital were 5.9 minutes (interquartile range (IOR) 3.3-8.9), 15.2 minutes (IOR 11.418.2) and 37.1 minutes (IOR 30.2-41.1) for the short, medium and long-distance groups, respectively.

\section{Prehospital mortality}

In all, 4133 patients with MI as the primary certified cause of death died without being hospitalised or before they reached a hospital (table 1). The age-adjusted death rate was $8.4 \%(8.0 \%$ for men and $9.3 \%$ for women); $50.9 \%$ of patients were women and $35.1 \%$ of patients lived $>9$ miles from the hospital. Compared with patients living $<3$ miles from hospitals those living $>3$ miles from hospital had higher MI mortality (adjusted OR $1.46,95 \%$ CI 1.09 to 1.95 for 3-9 miles and 2.05, 1.00 to 4.21 for $>9$ miles).

\section{Diagnostic accuracy of community $\mathrm{MI}$ death}

About $10 \%$ of patients certified as a community MI death had postmortem examinations. The postmortem results showed that MI was the cause of death in over $95 \%$ of these subjects during the study period.

\section{In-hospital mortality}

A total of 6408 patients (57.9\% male) survived to be hospitalised with incident acute MI during the study period and 1010 of these $(15.8 \%)$ died in hospital (that is, case fatality). The median duration of hospitalisation was 2 days. Except for $\beta$-blocker use patients who died in hospital had received more cardiovascular drug prescriptions in the previous year than patients who survived. There were significant differences in gender, age and social deprivation between patients who survived and those who died. Women and older patients had higher risk of mortality than men and young patients. The age-adjusted death rate was $11.6 \%$ (12.3\% for men and $10.8 \%$ for women). There were more women living closer to hospital than men. The percentages of women in each distance category were $36.2 \%$, 
Table 1 Characteristics of patients following myocardial infarction, Tayside

\begin{tabular}{|c|c|c|c|c|c|c|c|c|c|c|}
\hline & \multirow{2}{*}{\multicolumn{2}{|c|}{$\begin{array}{l}\text { Prehospital } \\
\text { Dead }\end{array}$}} & \multicolumn{4}{|c|}{ In-hospital } & \multicolumn{4}{|c|}{ Post-hospital } \\
\hline & & & \multicolumn{2}{|c|}{ Dead } & \multicolumn{2}{|l|}{ Alive } & \multicolumn{2}{|l|}{ Dead } & \multicolumn{2}{|l|}{ Alive } \\
\hline & No & $\%$ & No & $\%$ & No & $\%$ & No & $\%$ & No & $\%$ \\
\hline$<3$ & 1287 & 31.7 & 407 & 41.6 & 1753 & $33.0^{* *}$ & 678 & 36.5 & 1075 & $31.1^{* *}$ \\
\hline $3-9$ & 1346 & 33.2 & 304 & 31.0 & 1801 & 33.9 & 607 & 32.7 & 1194 & 34.6 \\
\hline$>8$ & 1423 & 35.1 & 268 & 27.4 & 1756 & 33.1 & 572 & 30.8 & 1184 & 34.3 \\
\hline Female & 2104 & 50.9 & 567 & 56.1 & 2128 & 39.4 & 890 & 46.7 & 1238 & 35.6 \\
\hline Age (mean, SD) & 77.2 & 10.9 & 78.5 & 9.6 & 67.5 & $12.6^{* *}$ & 74.6 & 10.2 & 63.7 & $12.1^{* *}$ \\
\hline \multicolumn{11}{|l|}{ Deprivation category } \\
\hline 1, least deprived & 175 & 4.4 & 47 & 4.8 & 313 & 5.9 & 100 & 5.4 & 213 & 6.1 \\
\hline 2 & 833 & 20.9 & 172 & 17.4 & 980 & 18.4 & 325 & 17.6 & 655 & 18.8 \\
\hline 3 & 1187 & 29.8 & 312 & 31.6 & 1508 & 28.3 & 502 & 27.3 & 1006 & 28.8 \\
\hline \multicolumn{11}{|l|}{ Urban-rural code } \\
\hline 1 & 1603 & 40.5 & 385 & 38.6 & 2356 & $44.0 * *$ & 813 & 43.5 & 1543 & 44.2 \\
\hline 2 & 1003 & 25.4 & 289 & 29.0 & 1410 & 26.3 & 527 & 28.2 & 883 & 25.3 \\
\hline 3,6 & 1181 & 29.9 & 280 & 18.0 & 1431 & 26.7 & 467 & 25.0 & 964 & 27.6 \\
\hline 7,8 & 167 & 4.2 & 44 & 4.4 & 163 & 3.0 & 62 & 3.3 & 101 & 2.9 \\
\hline \multicolumn{11}{|l|}{ Previous hospitalisation } \\
\hline Angina & 445 & 10.8 & 121 & 12.0 & 691 & 12.8 & 295 & 15.5 & 396 & $11.3^{* *}$ \\
\hline Heart failure & 845 & 20.5 & 277 & 27.4 & 1402 & $26.0^{* *}$ & 821 & 43.1 & 581 & $16.6^{* *}$ \\
\hline Stroke & 433 & 10.5 & 111 & 11.0 & 271 & $5.0^{* *}$ & 173 & 9.1 & 98 & $2.8^{* *}$ \\
\hline Hypertension & 432 & 10.5 & 131 & 13.0 & 645 & $12.0^{*}$ & 233 & 12.2 & 412 & $11.8^{* *}$ \\
\hline PVD & 462 & 11.2 & 128 & 12.7 & 423 & $7.8^{* *}$ & 232 & 12.2 & 191 & $5.5^{* *}$ \\
\hline Diabetes & 567 & 13.7 & 146 & 14.5 & 715 & 13.2 & 327 & 17.2 & 388 & $11.1^{* *}$ \\
\hline Nitrates & 1438 & 34.8 & 374 & 37.0 & 1627 & $30.1^{* *}$ & 1047 & 54.9 & 2435 & $69.8^{* *}$ \\
\hline Ca-blockers & 1370 & 33.2 & 370 & 36.6 & 1694 & 31.4 & 518 & 27.2 & 1177 & $33.7^{* *}$ \\
\hline
\end{tabular}

Data are numbers and percentages unless otherwise stated.

${ }^{*}$ Comparison of dead and alive patients, $\mathrm{p}<0.05 ;{ }^{* *} \mathrm{p}<0.01$. †Excluding missing data. $\$$ Drug uses during the follow-up for post-hospital cohort.

$A C E$, angiotensin-converting enzyme; PVD, peripheral vascular disease.

$33.1 \%$ and $30.6 \%$ for $<3$ miles, $3-9$ miles and $>9$ miles distance groups, respectively.

There was no increased risk of death in hospital with increasing distance between home and hospital of admission (table 2).

\section{Follow-up mortality}

In all, 5398 patients (3260 men, 2128 women) were discharged from hospital and were followed up for a median of 3.2 years (IOR 1.1-6.2). Of these, 1907 patients (35.3\%, 1017 men and 890 women) died during the follow-up period. The age-adjusted death rate was $8.6 \%$ ( $8.4 \%$ for men and $9.4 \%$ for women). There was a significantly increased use of statins and other cardiovascular drugs following hospitalisation compared with previous drug use (table 1). Patients who survived during follow-up had higher prescribing rates for cardiovascular drugs than patients who died (as we have shown before). ${ }^{10}$ A total of 3214 patients $(59.5 \%)$ were taking lipid-lowering drug treatment and, of these, 3194 (99.4\%) were taking statin treatment.
After adjustment for other risk factors, patients who lived $>9$ miles from the hospital had an increased risk of mortality during the follow-up period when compared to patients who lived $<3$ miles from the hospital (adjusted HR 1.90, 95\% CI 1.19 to 3.02) (table 2). A non-significant increased mortality was seen for those who lived 3-9 miles from the hospital (adjusted HR $1.27,95 \%$ CI 0.96 to 1.68 ).

\section{Effect of cardiovascular drugs}

Compared with non-users, users of lipid-lowering drugs (adjusted HR 0.47 , 95\% CI 0.42 to 0.53 ), antiplatelet drugs (adjusted HR $0.40,95 \%$ CI 0.35 to 0.45 ), $\beta$-blockers (adjusted HR $0.55,95 \%$ CI 0.49 to 0.62 ) and ACE inhibitors (adjusted HR $0.84,95 \%$ CI 0.75 to 0.93 ) during the follow-up had a significantly lower risk of mortality.

\section{Thrombolysis}

A total of 209 out of 5398 (3.8\%) patients had thrombylosis data for the follow-up analysis. This subgroup analysis suggested 
Table 2 Adjusted odds ratios (OR) or hazard ratios (HR) for mortality in patients with MI

\begin{tabular}{|c|c|c|c|}
\hline & Prehospital & In-hospital & Post-hospital \\
\hline & \multicolumn{3}{|c|}{ Number of event/total } \\
\hline \multicolumn{4}{|c|}{ Distance (miles), tertile* } \\
\hline$<3$ & $1287 / 3447$ & $407 / 2160$ & $678 / 1753$ \\
\hline $3-9$ & $1346 / 3451$ & $304 / 2105$ & $607 / 1801$ \\
\hline \multirow[t]{2}{*}{$>9$} & $\begin{array}{l}1423 / 3447 \\
\text { OR or HR }\end{array}$ & $268 / 2024$ & $572 / 1756$ \\
\hline & OR (95\% Cl) & OR $(95 \%$ Cl) & HR $(95 \%$ Cl) \\
\hline \multicolumn{4}{|c|}{ Unadjusted } \\
\hline$<3$ & 1.00 & 1.00 & 1.00 \\
\hline $3-9$ & 1.07 (0.97 to 1.18$)$ & $0.73(0.62$ to 0.86$)$ & $0.83(0.74$ to 0.92$)$ \\
\hline$>9$ & 1.18 (1.07 to 1.30$)$ & 0.66 (0.56 to 0.78$)$ & 0.88 (0.79 to 0.98$)$ \\
\hline \multicolumn{4}{|c|}{ Adjusted for age, sex and deprivation } \\
\hline$<3$ & 1.00 & 1.00 & 1.00 \\
\hline $3-9$ & $1.17(1.05$ to 1.30$)$ & $0.80(0.66$ to 0.95$)$ & $0.89(0.79$ to 1.00$)$ \\
\hline$>9$ & 1.20 (1.07 to 1.35$)$ & 0.63 (0.52 to 0.77 ) & $0.96(0.86$ to 1.10$)$ \\
\hline \multicolumn{4}{|c|}{ Adjusted for age, sex, deprivation and other covariates } \\
\hline$<3$ & 1.00 & 1.00 & 1.00 \\
\hline $3-9$ & $1.16(1.04$ to 1.29$)$ & 0.82 (0.68 to 0.98$)$ & $0.90(0.80$ to 1.01$)$ \\
\hline$>9$ & 1.18 (1.05 to 1.32$)$ & 0.66 (0.54 to 0.80$)$ & $0.97(0.85$ to 1.10$)$ \\
\hline \multicolumn{4}{|c|}{ Adjusted for age, sex, deprivation, other covariates and urban-rural code } \\
\hline$<3$ & 1.00 & 1.00 & 1.00 \\
\hline $3-9$ & 1.21 (1.07 to 1.37$)$ & 1.07 (0.73 to 1.58$)$ & $1.21(0.95$ to 1.54$)$ \\
\hline$>9$ & $1.30(1.10$ to 1.54$)$ & 0.79 (0.44 to 1.40$)$ & $1.49(1.04$ to 2.15$)$ \\
\hline \multicolumn{4}{|c|}{ Adjusted for age, sex, deprivation, other covariates and estimated travel time } \\
\hline$<3$ & 1.00 & 1.00 & 1.00 \\
\hline $3-9$ & 1.44 (1.16 to 1.78$)$ & 0.65 (0.44 to 0.98$)$ & $1.19(0.90$ to 1.58$)$ \\
\hline$>9$ & 2.37 (1.24 to 4.54$)$ & $1.04(0.22$ to 4.93$)$ & 2.06 (1.01 to 4.21$)$ \\
\hline \multicolumn{4}{|c|}{ Adjusted for age, sex, deprivation, other covariates, urban-rural code and estimated travel time } \\
\hline$<3$ & 1.00 & 1.00 & 1.00 \\
\hline $3-9$ & 1.46 (1.09 to 1.95$)$ & $1.02(0.66$ to 1.58$)$ & $1.27(0.96$ to 1.68$)$ \\
\hline$>9$ & 2.05 (1.00 to 4.21$)$ & 0.95 (0.45 to 2.03 ) & $1.90(1.19$ to 3.02$)$ \\
\hline
\end{tabular}

*0ther covariates included day of week of admission, previous cardiovascular disease, diabetes mellitus, cardiovascular drug use and interactions between distance and deprivation/sex. Analysis was done by excluding missing data.

that early thrombolysis reduced mortality (adjusted HR 0.63, $95 \%$ CI 0.37 to 1.07), However, early thrombolysis did not change the direction of the impact of distance (adjusted HR $1.86,95 \%$ CI 0.49 to 7.02 for patients who lived 3-9 miles from the hospital, adjusted HR $1.43,95 \%$ CI 0.18 to 11.39 for patients who lived $>9$ miles from the hospital when compared with patients who lived $<3$ miles from the hospital).

\section{DISCUSSION}

Previous studies have shown a relation between the treatment delay and mortality outcome in MI patients. ${ }^{11-14}$ However, few studies have investigated the impact of geographical location and none has been representative of a complete population. ${ }^{15}$ Our data suggest that the distance between home and hospital of admission predicts mortality in subjects experiencing first acute MI both in the community (prehospital) and following discharge from hospital after adjustment for urban-rural code or travel time and other covariates. There was no difference if these results were adjusted only for age, sex and other covariates, suggesting that urban-rural code and travel time are the significant confounders. Other areas that could cause a delay between the onset of symptoms and treatment are the delay by patients in calling for help and the delay between calling for help and the attendance of a doctor. We could not measure these but they could be investigated. ${ }^{16}$ However, we used a measure of rurality as a possible surrogate measure of patient behaviour in the different geographical settings.
The median travel time was 37 minutes for patients who lived $>9$ miles from an acute hospital. This time does not include time taken for the ambulance to reach the patient. In addition, these times take no account of adverse traffic or weather conditions. We think that it is unlikely that this group of patients could have met the national target for thrombolysis within 60 minutes of a call for help.

\section{Geographical inequality and early thrombolysis}

Our data support the concept that there is geographical inequality of care in patients who have had an acute incident MI. An excess in coronary heart disease (CHD) death rates in young patients was reported outside the state capital cities in Australia. ${ }^{17}$ The risk factors between the populations and inadequacies in the level of medical care provided were the two main reasons that have been used to explain this excess CHD death. A recent US study showed that a mile increase in distance leads to a nearly $6.5 \%$ increase in the number of deaths from a heart attack. ${ }^{18}$ However in Scotland, the National Health Service is tax funded, free at the point of consumption and covers the entire population. There should thus be no socioeconomic eligibility distinctions in the level of health care given to an individual, being based on need alone. One hypothesis is that the time delay related to distance plays an important part in the prognosis of MI patients. A study from our own region demonstrated that an early thombolysis treatment strategy was achievable and improved outcome. ${ }^{19}$ However when we 
adjusted for travel time this did not explain the association between distance from acute facilities and mortality.

A US Veteran Affairs (VA) study investigated the effect of distance between home and hospital of admission on mortality in male post-MI patients. ${ }^{15}$ They compared the mortality outcome between patients (64\%) who lived more than 20 miles from any VA health source and patients (36\%) who lived within 20 miles. They found a positive relation between distance and follow-up mortality. However they studied only men and their results may not be generalisable to other healthcare systems. Our study indicates that the distance from home to hospital of admission is an important factor that predicts survival following acute MI.

\section{Study limitations and strengths}

There are some limitations in our study. First, we did not have information on smoking, obesity, severity of MI, invasive management after discharge, the treating specialist at the admitting hospital and other unmeasured risk factors, all of which are likely to be important in patients with heart disease. However, we did use the Carstairs socioeconomic deprivation score as a surrogate measure for at least some of these factors. $^{2021}$ Also, our previous sensitivity analysis on unmeasured risk factors showed that at least some of these unknown risk factors were unlikely to make a big impact on cardiovascular outcome. ${ }^{22}$ Second, the distance we used in this study was based on home and hospital addresses. We did not know the exact location of the patients when they had a heart attack. However, given the average age of 72 , we would expect that the majority of patients would have had their MI at or near their home. Third, we did not have information on the time of onset of MI symptoms. Fourth, we used linear distance and estimated travel time in the study. However, distance along the road network may be different and there may have been differences in traffic conditions at different times of day.

We observed independent risk reductions in mortality during the follow-up period for lipid-lowering drugs, antiplatelet drugs and $\beta$-blockers consistent with the finding from clinical trials and other observational studies. ${ }^{23-28}$ However we may have underestimated the benefits of cardiovascular drugs because we assumed all patients complied with their drug treatment when in reality this is rarely the case.$^{28}$ Finally, the diagnostic accuracy of MI death before hospitalisation is open to question. The data retrieved from the General Register Office revealed that in those subjects who had postmortem examinations performed, the diagnosis of MI was almost always confirmed. Presumably those subjects who underwent post mortems were those in whom the diagnosis was in question and was more likely to be incorrect. Thus these postmortem data are reassuring and suggest that prehospital MI death data were reasonably accurate.

The strength of our study is the population-based cohort design, with complete follow-up over the study period. This approach allows a "real-world" population to be studied, representing all socioeconomic groups and within a universal healthcare coverage scheme. ${ }^{29}$ The results inform us that there may be geographical inequality in healthcare delivery, the cause of which is currently difficult to explain. This relation may influence healthcare policy on the provision of prehospital care such as thrombolysis for MI, the enhanced provision of paramedics and the greater delivery of ambulance services to remote communities. It is in line with the recent Kerr report in Scotland calling for services to be delivered closer to patients wherever possible, ${ }^{30}$ and studies demonstrating that managed clinical networks can achieve this goal. ${ }^{31}$

In conclusion, our data suggested that distance from home to hospital may predict mortality outcome in subjects experiencing an acute MI. These data provide support for policies that locate services for acute MI closer to where patients live.

Funding: LW holds a Special Training Fellowship in Health Services and Health of the Public Research Award from the MRC, UK

Competing interests: None.

Ethical approval: This study was approved by Tayside Research Ethics Committee and the Tayside Caldicott Guardians.

\section{REFERENCES}

1. Department of Health. National service framework for coronary heart disease. London: DH 2000. http://www.dh.gov.uk/assetRoot/04/05/75/26/04057526.pdf (accessed 29 May 2008).

2. Morrison LJ, Verbeek PR, McDonald AC, et al. Mortality and prehospital thrombolysis for acute myocardial infarction: a meta-analysis. JAMA 2000;283:2686-92.

3. Scottish Government. Delivering for health. http://www.scotland.gov.uk/ Publications/2005/11/02102635/26356 (accessed 29 May 2008).

4. Scottish Government. Building a health service fit for the future http://www. scotland.gov.uk/Publications/2005/05/23141307/13135 (accessed 29 May 2008)

5. Wei L, Parkinson J, MacDonald TM, The Tayside Medicines Monitoring Unit (MEM0). In: Strom BL, ed. Pharmacoepidemiology. 4th ed. Chichester: John Wiley, 2005:323-36.

6. Donnan PT, Dougall HT, Sullivan F. Optimal strategies for identifying patients with myocardial infarction in general practice. Family Practice 2003;20:706-10.

7. Scottish Government. Scottish health survey, 2003. http://www.scotland.gov.uk/ Publications/2005/11/25145024/50253 (accessed 29 May 2008)

8. Carstairs V. Deprivation and health in Scotland. Health Bull (Edinb) 1990:48:162-75

9. Evans C, Chalmers J, Capewell S, et al. "I don't like Mondays" — day of the week of coronary heart disease deaths in Scotland: study of routinely collected data. BMJ 2000:320:218-9.

10. Wei L, Ebrahim S, Bartlett C, et al. Cohort study of statin use in the secondary prevention of coronary heart disease in primary care: comparison of inclusion and outcome with trial data. BMJ 2005;330:821-4.

11. Rogers WJ, Canto JG, Lambrew CT, et al. Temporal trends in the treatment of over 1.5 million patients with myocardial infarction in the US from 1990 through 1999: the National Registry of Myocardial Infarction 1,2 and 3. J Am Coll Cardiol 2000;36:2056-63

12. Goldberg RJ, Steg PG, Sadiq I, et al. Extent of, and factors associated with, delay to hospital presentation in patients with acute coronary disease (the GRACE registry). Am J Cardiol 2002;89:791-6.

13. Rawles J. Magnitude of benefit from earlier thrombolytic treatment in acute myocardial infarction: new evidence from Grampian region early anistreplase tria (GREAT). BMJ 1996;312:212-5.

14. Capewell S, Maclntyre K, Stewart S, et al. Age, sex, and social trends in out-ofhospital cardiac deaths in Scotland 1986-95: a retrospective cohort study. Lancet 2001;358:1213-7.

15. Piette JD, Moos RH. The influence of distance on ambulatory care use, death, and readmission following a myocardial infarction. Health Serv Res 1996;31:573-91.

16. Robertson R, Campbell NC, Smith S, et al. Factors influencing time from presentation to treatment of colorectal and breast cancer in urban and rural areas. Br J Cancer 2004;90:1479-85.

17. Sexton PT, Sexton TL. Excess coronary mortality among Australian men and women living outside the capital city statistical divisions. Med J Aust 2000;172:370-4.

18. Buchmueller TC, Jacobson M, Wold C. How far to the hospital? The effect of hospital closures on access to care. J Health Econ 2006;25:740-61.

19. Pedley DK, Bissett K, Connolly EM, et al. Prospective observational cohort study of time saved by prehospital thrombolysis for ST elevation myocardial infarction delivered by paramedics. BMJ 2003;327:22-6.

20. Scottish Government. Scottish health survey 1995. Volume 1, chapter 4: Smoking Edinburgh: Stationery Office 1997:140-1.

21. Evans JM, Newton RW, Ruta DA, et al. Socio-economic status, obesity and prevalence of type 1 and type 2 diabetes mellitus. Diabet Med 2000;17:478-80.

22. Wei L, MacDonald TM, Walker BR. Taking glucocorticoids by prescription is associated with subsequent cardiovascular disease. Ann Intern Med 2004; 141:764-70.

23. Pedersen TR, Kjekshus J, Berg K, et al. Randomised trial of cholesterol lowering in 4444 patients with coronary heart disease: the Scandinavian Simvastatin Survival Study (4S). Lancet 1994:344:1383-9.

24. Sacks FM, Pfeffer MA, Moye LA, et al. The effect of pravastatin on coronary events after myocardial infarction in patients with average cholesterol levels. Cholesterol and Recurrent Events Trial investigators. N Engl J Med 1996;335:1001-9.

25. Tonkin A, Aylward P, Colquhoun D, et al. Prevention of cardiovascular events and death with pravastatin in patients with coronary heart disease and a broad range of initial cholesterol levels. N Engl J Med 1998;339:1349-57. 
26. Gottlieb SS, McCarter RJ, Vogel RA. Effect of beta-blockade on mortality among high-risk and low-risk patients after myocardial infarction. N Engl J Med 1998;339:489-97.

27. Baigent C, Sudlow C, Collins R, et al. Collaborative meta-analysis of randomised trials of antiplatelet therapy for prevention of death, myocardial infarction, and stroke in high risk patients. BMJ 2002;324: 71-86.

28. Wei L, Flynn R, Murray GD, et al. Use and adherence to beta-blockers for secondary prevention of myocardial infarction: who is not getting the treatment. Pharmacoepidemiol Drug Saf 2004;13:761-6.
29. MacDonald TM, Morant SV, Robinson GC, et al. Association of upper gastrointestinal toxicity of non-steroidal anti-inflammatory drugs with continued exposure: cohort study. BMJ 1997;315:1333-7.

30. Report calls for more community based health care in Scotland. http://bmi. bmjjournals.com/cgi/content/full/330/7503/1288-c and http://www.scotland.gov.uk/ Publications/ (accessed 29 May 2008).

31. Hamilton KE, Sullivan FM, Donnan PT, et al. A managed clinical network for cardiac services: set-up, operation and impact on patient care. Int J Integr Care 2005:5:1-15.

\section{Save your favourite articles and useful searches}

Use the "My folders" feature to save and organise articles you want to return to quickly-saving space on your hard drive. You can also save searches, which will save you time. You will only need to register once for this service, which can be used for this journal or all BMJ Journals, including the BMJ. 